\title{
Final results from the Palo Verde neutrino oscillation experiment
}

\author{
F. Boehm, ${ }^{3}$ J. Busenitz, ${ }^{1}$ B. Cook,${ }^{3}$ G. Gratta, ${ }^{4}$ H. Henrikson, ${ }^{3}$ J. Kornis, ${ }^{1}$ D. Lawrence, ${ }^{2}$ K. B. Lee, ${ }^{3}$ K. McKinny, ${ }^{1}$ \\ L. Miller, ${ }^{4}$ V. Novikov, ${ }^{3}$ A. Piepke, ${ }^{1,3}$ B. Ritchie, ${ }^{2}$ D. Tracy, ${ }^{4}$ P. Vogel, ${ }^{3}$ Y-F. Wang, ${ }^{4}$ and J. Wolf ${ }^{1}$ \\ ${ }^{1}$ Department of Physics and Astronomy, University of Alabama, Tuscaloosa, Alabama 35487 \\ ${ }^{2}$ Department of Physics and Astronomy, Arizona State University, Tempe, Arizona 85287 \\ ${ }^{3}$ Division of Physics, Mathematics and Astronomy, Caltech, Pasadena, California 91125 \\ ${ }^{4}$ Physics Department, Stanford University, Stanford, California 94305
}

(Received 3 July 2001; published 5 November 2001)

\begin{abstract}
The analysis and results are presented from the complete data set recorded at Palo Verde between September 1998 and July 2000. In the experiment, the $\bar{\nu}_{\mathrm{e}}$ interaction rate has been measured at a distance of 750 and 890 $\mathrm{m}$ from the reactors of the Palo Verde Nuclear Generating Station for a total of 350 days, including 108 days with one of the three reactors off for refueling. Backgrounds were determined by (a) the swap technique based on the difference between signal and background under reversal of the positron and neutron parts of the correlated event, and (b) making use of the conventional reactor-on and reactor-off cycles. There is no evidence for neutrino oscillation and the mode $\bar{\nu}_{\mathrm{e}} \rightarrow \bar{\nu}_{x}$ was excluded at $90 \%$ C.L. for $\Delta m^{2}>1.1 \times 10^{-3} \mathrm{eV}^{2}$ at full mixing, and $\sin ^{2} 2 \theta>0.17$ at large $\Delta m^{2}$.
\end{abstract}

DOI: 10.1103/PhysRevD.64.112001

PACS number(s): 13.15.+g, 14.60.Lm, 14.60.Pq

\section{INTRODUCTION}

Here we report the final results of a long baseline study of $\bar{\nu}_{\mathrm{e}}$ oscillations at the Palo Verde Nuclear Generating Station. This is a continuation of the work reported earlier in Refs. $[1,2]$, in which details of the experiment and first results were described. Hence we only briefly describe the detector and the analysis, stressing the improvements and final results. Since the previous report, the data sample has been almost doubled. Improvements have been made on reconstruction and simulation, reducing the systematic error by one-third.

The experiment was originally motivated by the observation of an anomalous atmospheric neutrino ratio $\nu_{\mu} / \nu_{\mathrm{e}}$ reported in several independent experiments [3-5]. The mass parameter suggested by this anomaly is in the range of $10^{-2}<\Delta m^{2}<10^{-3} \mathrm{eV}^{2}$ for two-flavor neutrino oscillation. The Palo Verde experiment, together with the CHOOZ experiment $[6,7]$ with a similar baseline, were able to exclude $\nu_{\mu} \rightarrow \nu_{\mathrm{e}}$ oscillations as the dominant mechanism for the atmospheric neutrino anomaly. While the experiment has pursued its goal of exploring the then unknown region of small $\Delta m^{2}$, recent data from Super-Kamiokande [8] favor the $\nu_{\mu}$ $\rightarrow \nu_{\tau}$ oscillation channel over the $\nu_{\mu} \rightarrow \nu_{\mathrm{e}}$ channel.

\section{EXPERIMENTAL TECHNIQUE}

\section{A. Detector and signal}

The experiment was performed at the Palo Verde Nuclear Generating Station in Arizona. The plant consists of three identical pressurized water reactors with a total thermal power of $11.63 \mathrm{GW}$. The detector was located at a shallow underground site, $890 \mathrm{~m}$ from two of the reactors and $750 \mathrm{~m}$ from the third. The 32 meter-water-equivalent overburden entirely eliminated any hadronic component of cosmic radiation and reduced the cosmic muon flux.
The segmented detector [2] consisted of 66 acrylic cells filled with 11.34 tons of Gd-loaded liquid scintillator [9]. A $0.8 \mathrm{~m}$ long oil buffer at both ends of each $9 \mathrm{~m}$ long cell and a $1 \mathrm{~m}$ buffer filled with water (105 t) surrounding the central detector shielded it from radioactivity originating in the photomultiplier tubes (PMT's) and laboratory walls as well as from neutrons produced by cosmic muons passing outside of the detector. The outermost layer of the detector was an active muon veto counter, providing $4 \pi$ coverage. All materials used in the construction of the detector and the laboratory were screened for their radioactivity content by means of low background gamma ray spectroscopy in order to control detector background.

The $\bar{\nu}_{\mathrm{e}}$ flux was detected via the correlated positron and neutron subevents from the reaction $\bar{\nu}_{\mathrm{e}} \mathrm{p} \rightarrow \mathrm{ne}^{+}$. The subevents are (1) the positron's kinetic energy $(\langle E\rangle$ $\simeq 2.4 \mathrm{MeV}$ ) and two prompt annihilation $\gamma$ 's, and (2) the subsequent (delayed with a time constant of $\sim 27 \mu \mathrm{s}) \quad \gamma$ 's from capture of the thermalized neutron on Gd (with energy $\sim 8 \mathrm{MeV}$ ).

The data acquisition electronics was built as a dual bank system, allowing both parts of the sequential $\bar{\nu}_{\mathrm{e}}$ capture event to be recorded with no deadtime by switching between banks. Signals from each PMT were discriminated by two thresholds: a high threshold corresponding to $\sim 600 \mathrm{keV}$ for energy deposits in the middle of the cell and a low threshold corresponding to $\sim 40 \mathrm{keV}$ also in the middle of the cell, or a single photoelectron at the PMT. The trigger processor, a field programmable gate array, searched for triple patterns in the central detector for each of the subevents, requiring one high discriminator signal and at least two low discriminator signals from neighboring cells [10]. All events with two triple signals within $450 \mu \mathrm{s}$ of each other were written to disk.

A veto signal following the passage of a muon (typical veto rates were $\sim 2 \mathrm{kHz}$ ) disabled the central detector trigger for $10 \mu \mathrm{s}$. With each event, the time and hit pattern of 
the previous muon in the veto counter was recorded for offline use along with information as to whether or not the muon passed through the target cells. The veto inefficiency was measured to be $2.5 \pm 0.2 \%$ for stopping muons (one hit missed) and $0.07 \pm 0.02 \%$ for through-going muons (two hits missed).

In order to maintain constant data quality during running, a protocol of continuous calibration and monitoring of all central detector cells was followed. Relative timing and position were calibrated with blue light emitting diodes (LED's) installed inside each cell. Additional blue LED's illuminated optical fibers at the end of each cell, providing information about PMT linearity and short term gain changes. LED and fiber optic scans were performed once a week. For absolute energy calibration and determination of the positron and neutron detection efficiencies, as well as mapping of the light attenuation in each cell, radioactive sources were used. A complete source scan was undertaken every 2-3 months. Further details were described in Ref. [2].

\section{B. Expected $\bar{\nu}_{\mathrm{e}}$ interaction rate}

To evaluate the expected $\bar{\nu}_{\mathrm{e}}$ interaction rate in the detector, the power and fuel composition of the three reactors must be known. The calorimetric methods, based on the measurement of temperature and water flow rate in the secondary cooling loop, provided a power determination with $0.7 \%$ uncertainty.

The fission rates in the three reactor cores were calculated daily using a simulation code provided by the manufacturer of the reactors. The output of the core simulation was checked by measuring isotopic abundances in expended fuel elements in the core; errors in fuel exposure and isotopic abundances are estimated to cause $<0.3 \%$ uncertainty in the $\bar{\nu}_{\mathrm{e}}$ flux estimate. Four isotopes- ${ }^{239} \mathrm{Pu},{ }^{241} \mathrm{Pu},{ }^{235} \mathrm{U}$, and ${ }^{238} \mathrm{U}$ - produce virtually all the thermal power as well as all the $\bar{\nu}_{\mathrm{e}}$ 's. Measurements of the neutrino yield per fission and energy spectra exist for the first three isotopes [11,12]. The ${ }^{238} \mathrm{U}$ yield, which contributes $11 \%$ to the final $\bar{\nu}_{\mathrm{e}}$ rate, was calculated from theory [13] with an uncertainty of 10\%. The contribution of ${ }^{238} \mathrm{U}$ to the uncertainty of the total neutrino rate is therefore $\sim 1 \%$.

The $\bar{\nu}_{\mathrm{e}}$ energy spectrum was reconstructed from the measured positron kinetic energy. The approximate relation $E_{\bar{\nu}_{\mathrm{e}}}$ $=E_{e^{+}}+1.8 \mathrm{MeV}$ is slightly modified by the kinetic energy carried away by the neutron $(\sim 50 \mathrm{keV})$. The cross section of the detection reaction is accurately known [14]; the dominant uncertainty $(0.2 \%)$ stems from the neutron lifetime.

Previous short baseline reactor experiments have found good agreement between calculated and observed neutrino fluxes [15-17]. In particular, Ref. [17] quoted an uncertainty in the neutrino flux per fission of $1.4 \%$. Together with the combined uncertainty of $1.5 \%$ of the reactor power, the distance to the detector and the number of target atoms, the total systematic uncertainty of the $\bar{\nu}_{e}$ interaction rate therefore amounts to $2.1 \%$.

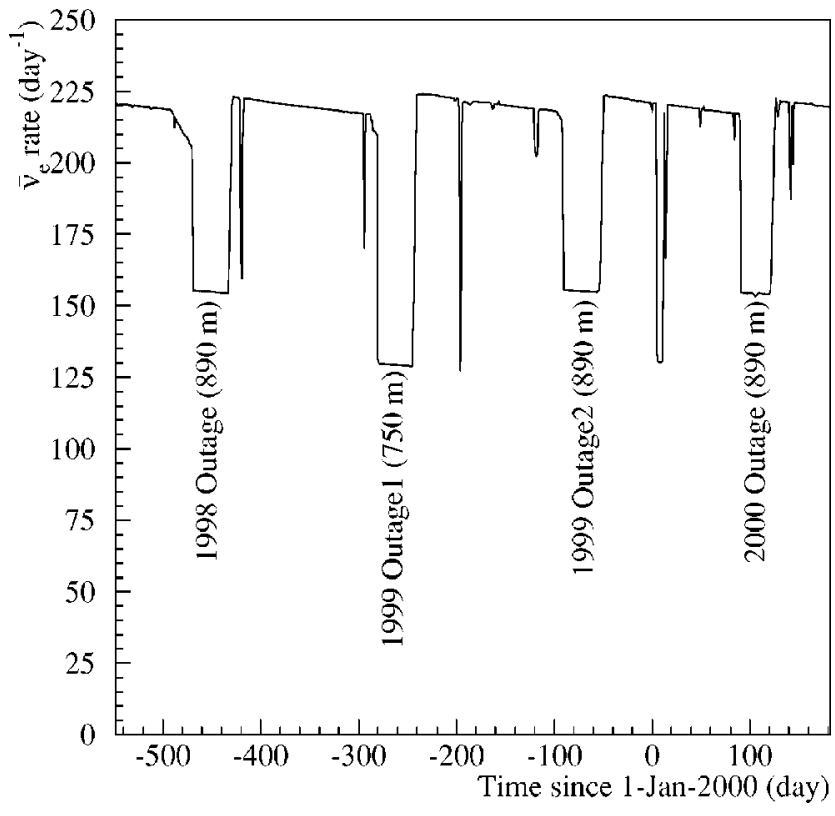

FIG. 1. The calculated $\bar{\nu}_{\mathrm{e}}$ interaction rate in the detector target for the case of no oscillations. Four long periods of reduced flux from reactor refuelings were used for background subtraction. The decreasing rate during the full power operation is a result of the changing core composition as the reactor fuel is burned.

The expected $\bar{\nu}_{\mathrm{e}}$ interaction rate in the whole target, both scintillator and the acrylic cells, is plotted in Fig. 1 for the case of no oscillation from July 1998 to July 2000. Around 220 interactions per day are expected with all three units at full power. Four periods of sharply reduced rate occurred when one of the three reactors was off for refueling, the more distant reactors contributing each approximately $30 \%$ of the rate and the closer reactor the remaining $40 \%$. The short spikes of decreased rate are due to accidental reactor outages, usually less than a day. The gradual decline in rate between refuelings is caused by fuel burn-up, which changes the fuel composition in the core and the relative fission rates of the isotopes, thereby affecting slightly the yield and spectral shape of the emitted $\bar{\nu}_{\mathrm{e}}$ flux.

\section{MONTE CARLO SIMULATION}

\section{A. Detection efficiency}

An accurate understanding of the $\bar{\nu}_{\mathrm{e}}$ efficiency is crucial. Therefore, as described in Ref. [2], two parallel and independent event reconstruction and detector simulation Monte Carlo codes have been developed. Both give consistent results; in Ref. [2] the reported results were based on one of them, here most of the results are based on the second method.

A Monte Carlo model with a detailed simulation of the detector response, including the PMT pulse shape, is essential to simulate the rather strong dependence of the $\bar{\nu}_{\mathrm{e}}$ efficiency on the event location in the detector and, to a lesser extent, on time due to some scintillator aging. A variety of measurements was performed to cross check the Monte Carlo modeling of the detector response. 
The simulation code [2] contains the whole detector geometry and simulates the energy, time, and position of energy deposits in the detector using GEANT 3.21 [18]. GFLUKA [19] is used to simulate hadronic interactions, while for the low energy neutron transport GCALOR [20] is employed. Scintillator light quenching, parametrized as a function of ionization density, is included in the simulation [21].

Given the output of the physics generators, the Monte Carlo simulates the detector response in the form of PMT pulses which are converted into time and amplitude digitizations and trigger hits. Digitized data are then reconstructed with the same programs as real data, providing the trigger and selection cuts efficiencies.

\section{B. Improvements of the simulation}

Since the initial results were published [2], the data sample has been almost doubled. There were also improvements in the analysis due to refinements of the simulation of the detector response. Three changes had the largest impact on the quality of the simulation:

The pulse shapes of several hundred single-photoelectron (SPE) signals were digitized and compared with each other. An average SPE pulse shape was deduced, replacing the simple model that used only fixed rise and decay times.

The scintillation light was traced through the cell to the PMT's. SPE pulse shapes with constant charge-to-amplitude ratios were added up to the final PMT pulse for each photoelectron produced in the cathode. The trigger threshold was compared to the amplitude of the total PMT pulse. However, the charge-to-amplitude ratio of the measured SPE pulses varied slightly from pulse to pulse, resulting in a smeared trigger threshold when plotted as a function of ADC counts. Instead of varying the width of the average SPE pulse in the simulation, the relative height of the threshold was sampled from a Gaussian distribution with $\mu=1$ and $\sigma$ adjusted to describe the measured slope of the trigger efficiency versus ADC counts. The resulting modeling improvements can be recognized by comparing the trigger threshold accuracy in Fig. 2 with the analogous quantities in Fig. 9 of Ref. [2]).

The constant thresholds used for all cells and runs were replaced by an individual threshold for each discriminator. Variations in time were also taken into account by tracking thresholds using neutrino runs.

In conclusion the new simulation reduced the spread of data/simulation over all cells in the detector (Fig. 2) from $19.2 \%$ to $10.2 \%$ for the low (SPE) threshold and from $7.6 \%$ to $3.5 \%$ for the high threshold.

\section{Test of the $\bar{\nu}_{\mathrm{e}}$ detection efficiency}

${ }^{22} \mathrm{Na}$ and $\mathrm{Am}-\mathrm{Be}$ sources were used to verify the absolute efficiency of the detector for positron annihilations and subsequent neutron captures. The $1.275 \mathrm{MeV}$ primary $\gamma$ of the ${ }^{22} \mathrm{Na}$ source is accompanied $90 \%$ of the time by a low energy positron which annihilates in the source capsule. The primary $\gamma$ mimics the positron ionization associated with a low energy $\bar{\nu}_{\mathrm{e}}$ event and, together with the annihilation $\gamma$ 's, closely approximates the positron portion of a $\bar{\nu}_{\mathrm{e}}$ event near the trigger threshold.
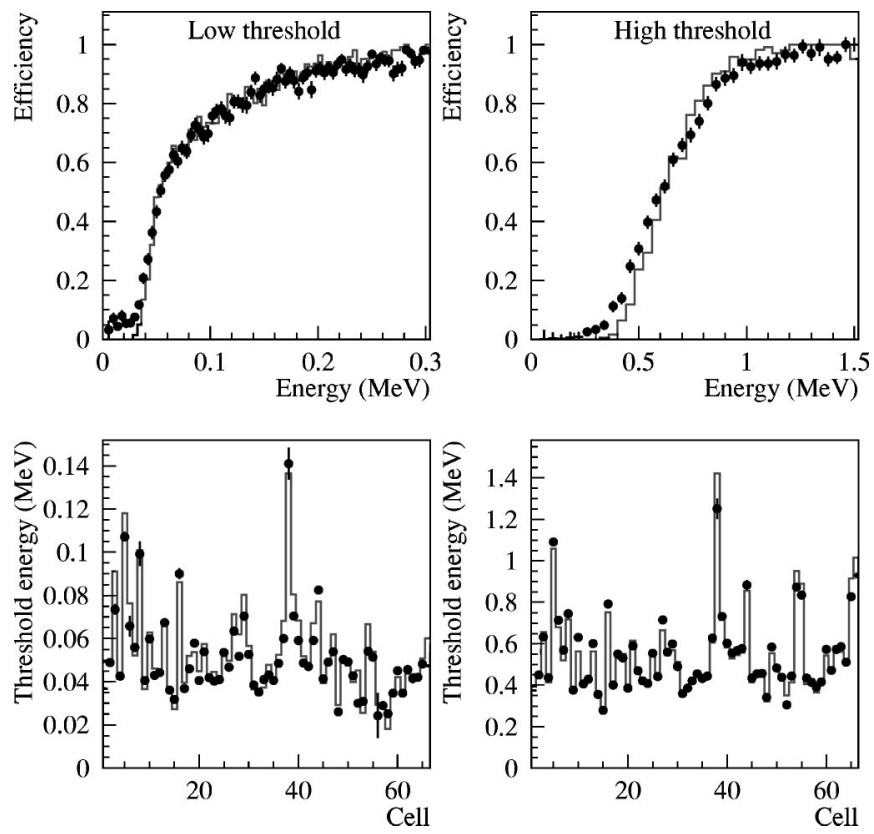

FIG. 2. The upper plots show the simulated and measured trigger efficiency for low and high thresholds as a function of energy deposited in the center of one cell. Dots represent data, while the solid line shows the simulated efficiency. The lower plots show the energy corresponding to a trigger efficiency of $50 \%$ for each cell. The spread between data and the Monte Carlo simulation has been improved by a factor of about 2 compared to Ref. [2].

The ${ }^{22} \mathrm{Na}$ source was inserted into the central detector at various locations during four dedicated calibration periods, separated by several months. A total of 85 different runs were taken in order to sample various distances from the PMT's and edges of the fiducial volume. This allows determination of an absolute efficiency, since the source activity is known to $1.5 \%$. After applying loose cuts to suppress background and correcting for detector deadtime, the measured absolute trigger efficiency could be compared with the Monte Carlo prediction; the results are shown in the top panel of Fig. 3. Good agreement is seen in the average efficiency over all runs (the spread in data and Monte Carlo simulations has been improved to $6.7 \%$ in 85 locations compared to $11.1 \%$ in 36 locations in Ref. [2]), and the agreement between the four calibration periods was better than $1.4 \%$. The ${ }^{22} \mathrm{Na}$ energy spectra predicted by the simulation and measured in the data also agree well. This comparison tests all aspects of the simulations: the high and low trigger thresholds, and the total energy deposit.

In order to check the neutron capture detection efficiency, the Am-Be neutron source was attached to one end of a thin $(7.5 \mathrm{~mm}) \mathrm{NaI}(\mathrm{Tl})$ detector readout by a flat PMT, so that the entire assembly could be still inserted in the gaps between each cell and its neighbor above or below. The $\mathrm{NaI}(\mathrm{Tl})$ detector tagged the $4.4 \mathrm{MeV} \gamma$ emitted in coincidence with a neutron. The $\mathrm{NaI}(\mathrm{Tl})$ tag forced the digitization of the 4.4 $\mathrm{MeV} \gamma$ as the prompt part of an event and opened a $450-\mu \mathrm{s}$ window for neutron capture, the same coincidence window as used in the $\bar{\nu}_{\mathrm{e}}$ runs. 

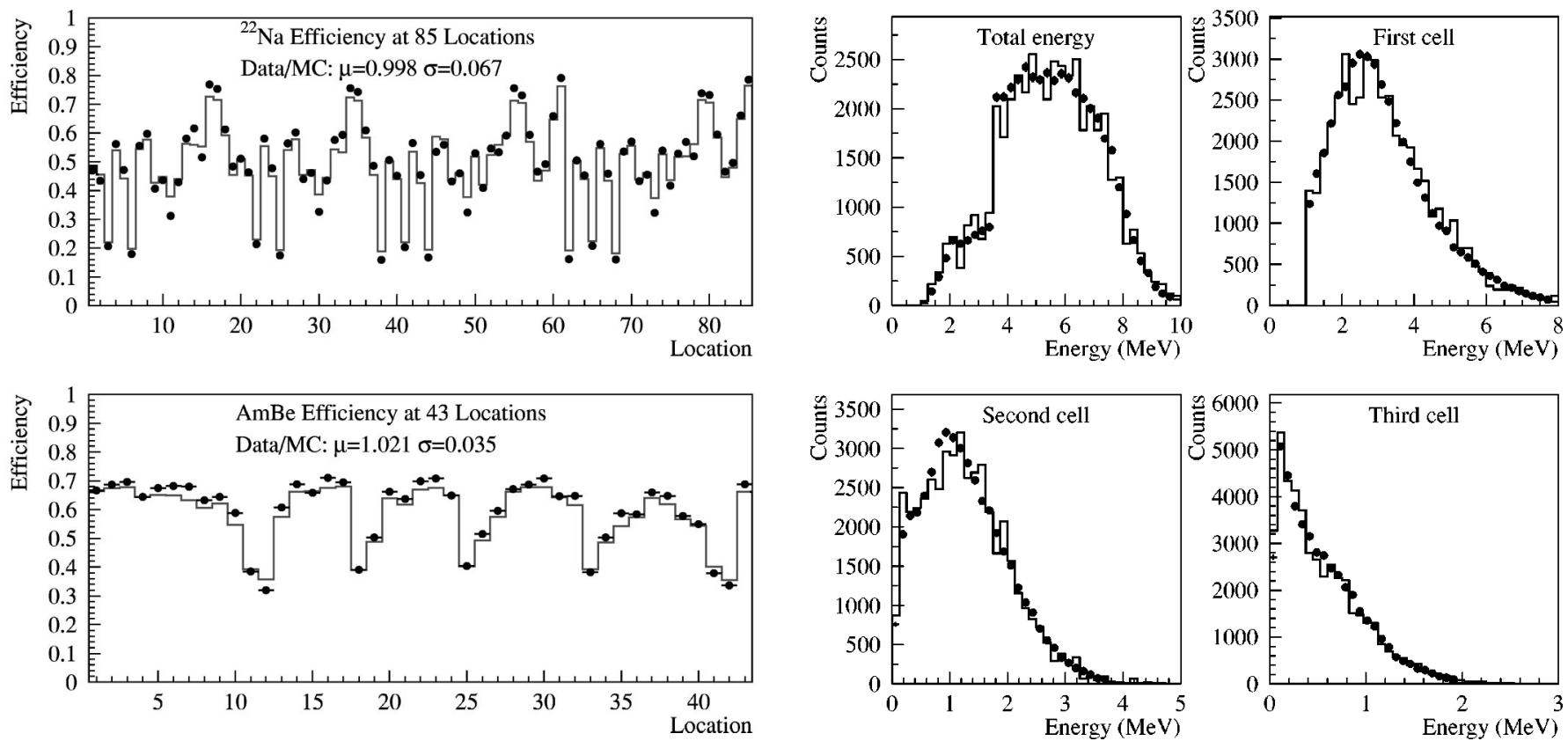

FIG. 3. Comparison of data (points) and the Monte Carlo simulations (histograms) for detection efficiency for ${ }^{22} \mathrm{Na}$ and $\mathrm{Am}-\mathrm{Be}$ source runs at various locations. For positions of the radioactive source near the border of the central detector we measure lower efficiencies in good agreement with the simulation (see locations $3,5,22, \ldots$ for ${ }^{22} \mathrm{Na}$ or locations $11,12,18 \ldots$ for $\left.\mathrm{Am}-\mathrm{Be}\right)$.

Loose neutron cuts were applied, and corrections were made for detector deadtime and a low rate of random background. On average, the Monte Carlo efficiency predictions agree well over the 43 locations tested (compared to 25 locations in Ref. [2]) with an average agreement of better than $2.1 \%$, as shown in the lower panel of Fig. 3. Small adjustments of parameters of the detector simulations could improve the agreement of the AmBe efficiencies, but at the same time led to larger disagreement for other parameters, e.g., ${ }^{22} \mathrm{Na}$ efficiencies or the shape of energy spectra. Therefore, only directly measured parameters (trigger efficiency as a function of charge and shape of PMT pulses) were used to adjust Monte Carlo parameters. Measured efficiencies for ${ }^{22} \mathrm{Na}$ and $\mathrm{AmBe}$ were only used to estimate the systematic uncertainty of the simulation.

Again, the energy spectra for neutrons predicted by the simulation and measured in the data were compared. The total energy seen in all cells and the energy detected in the three most energetic hits is plotted in Fig. 4. This test was done with cosmic muon induced neutrons, which are the dominant type of correlated events in neutrino runs. The neutrons were equally distributed over the whole detector.

These procedures completely test our $\bar{\nu}_{\mathrm{e}}$ efficiency simulation. Thus our ability to accurately generate the events, model the detector response, reconstruct the events, and correctly calculate the lifetime of the data acquisition (DAQ) system was verified.

The Monte Carlo simulation yielded an average efficiency over the whole detector as a function of $\bar{\nu}_{\mathrm{e}}$ energy. The simulation included interactions in the acrylic walls of the cells, since there is significant efficiency for inverse beta decay

FIG. 4. Comparison of data (points) and Monte Carlo simulation (histograms) for the spectra of total energy and first, second, and third most energetic hit ( $E_{\text {total }}, E_{1}, E_{2}$, and $\left.E_{3}\right)$ for capture cosmic muon induced neutrons. The sharp feature at $3.5-\mathrm{MeV}$ total energy is related to the requirement that at least one sub-event has a total energy above this value (see the text).

originating there. Next, the efficiency from the simulation was folded with the incident $\bar{\nu}_{\mathrm{e}}$ spectrum (including possible distortions due to oscillations) to obtain the overall efficiency which generally depends on the oscillation parameters $\Delta m^{2}$ and $\sin ^{2} 2 \theta$.

\section{ANALYSIS AND RESULTS}

Here we briefly discuss event reconstruction, event selection, efficiencies, and backgrounds. Details may be found in Refs. [1,2]. Both the analysis presented here and the one used for our previous papers were repeated without changing selection cuts for the present data-set.

The energies and positions associated with hits were reconstructed for each bank. The position of the hit along the length of the cell was determined from TDC times with a time-walk correction applied on the basis of the collected charge. The collected charge for each end was corrected for light attenuation and PMT nonlinearity and converted to an energy using energy calibration constants. The hit energy was determined as the weighted average of the measurements from either end.

To select events in the energy ranges where the triggers are efficient, we required that each sub-event (prompt "positron" and delayed "neutron") have at least one hit greater than $1 \mathrm{MeV}$ and at least two additional hits with energy greater than $30 \mathrm{keV}$. Any event with hits greater than $8 \mathrm{MeV}$ in either sub-event was discarded. The magnitude and pattern of energy deposits in the prompt sub-event were required to resemble what was expected from the kinetic energy of the positron and its annihilation. (The annihilation $\gamma$ 's each had 
TABLE I. Data taking periods, efficiencies (including lifetime), measured event rates $N_{1}$ and $N_{2}$, and results of the swap analysis (see the text), including the various background estimates. Uncertainties are statistical only.

\begin{tabular}{|c|c|c|c|c|c|c|c|c|}
\hline \multirow{2}{*}{$\begin{array}{l}\text { Period } \\
\text { Reactor }\end{array}$} & \multicolumn{2}{|c|}{1998} & \multicolumn{2}{|c|}{ 1999-I } & \multicolumn{2}{|c|}{ 1999-II } & \multicolumn{2}{|c|}{2000} \\
\hline & on & $890 \mathrm{~m}$ off & on & $750 \mathrm{~m}$ off & on & $890 \mathrm{~m}$ off & on & $890 \mathrm{~m}$ off \\
\hline time (days) & 30.4 & 29.4 & 68.2 & 21.8 & 60.4 & 29.6 & 83.2 & 27.5 \\
\hline \multirow[t]{2}{*}{ efficiency $(\%)$} & 8.0 & 8.0 & 11.5 & 11.6 & 11.6 & 11.6 & 10.9 & 10.8 \\
\hline & \multicolumn{8}{|c|}{ measured rates } \\
\hline$N_{1}\left(\mathrm{~d}^{-1}\right)$ & $39.6 \pm 1.1$ & $34.8 \pm 1.1$ & $54.9 \pm 0.9$ & $45.1 \pm 1.4$ & $54.2 \pm 0.9$ & $49.4 \pm 1.3$ & $52.9 \pm 0.8$ & $43.1 \pm 1.3$ \\
\hline$N_{2}\left(\mathrm{~d}^{-1}\right)$ & $25.1 \pm 0.9$ & $21.8 \pm 0.9$ & $33.4 \pm 0.7$ & $32.0 \pm 1.2$ & $32.5 \pm 0.7$ & $32.6 \pm 1.0$ & $30.2 \pm 0.6$ & $30.4 \pm 1.1$ \\
\hline \multirow[t]{2}{*}{$\left(1-\epsilon_{1}\right) B_{\mathrm{pn}}\left(\mathrm{d}^{-1}\right)$} & 0.88 & 0.89 & 1.11 & 1.11 & 1.11 & 1.11 & 1.07 & 1.07 \\
\hline & \multicolumn{8}{|c|}{ efficiency corrected rates } \\
\hline Background $\left(\mathrm{d}^{-1}\right)$ & $292 \pm 11$ & $255 \pm 10$ & $265 \pm 6$ & $266 \pm 10$ & $256 \pm 6$ & $265 \pm 9$ & $249 \pm 5$ & $272 \pm 9$ \\
\hline$R_{\nu}\left(\mathrm{d}^{-1}\right)$ & $202 \pm 19$ & $182 \pm 18$ & $212 \pm 10$ & $124 \pm 17$ & $214 \pm 11$ & $161 \pm 15$ & $237 \pm 10$ & $129 \pm 16$ \\
\hline$R_{\text {calc }}\left(\mathrm{d}^{-1}\right)$ & 216 & 154 & 218 & 129 & 220 & 155 & 218 & 154 \\
\hline
\end{tabular}

to have energy less than $600 \mathrm{keV}$, and together less than 1.2 $\mathrm{MeV}$. This is the only cut which treats the two sub-events asymmetrically.) The prompt and delayed sub-events were required to be correlated in space and time. To further suppress backgrounds, an event was accepted if it started at least $150 \mu \mathrm{s}$ after the last veto hit and at least $3.5 \mathrm{MeV}$ of energy was deposited in either the prompt or delayed sub-event.

The event yield must then be corrected for the efficiency of trigger and selection cuts as well as for detector deadtime, which has two components. The first one is the loss of neutrino events due to muons crossing the detector (a) within $150 \mu$ s before the start of the neutrino event or (b) between the prompt and delayed subevents. Its magnitude was determined from the measured muon veto rate and the distribution of inter-event times from detector simulation. The experiment lifetime after losses due to the muon veto is approximately $66 \%$. The second deadtime component comes from the DAQ system being unavailable to digitize a triple. Its magnitude is the ratio of the number of triples for which the DAQ was busy to the total number of triples "seen" by the trigger which could be directly measured using scalers. The deadtime of the trigger itself was measured to be less than $0.1 \%$. The experimental DAQ lifetime was about $81 \%$ for 1998 and $92 \%$ for 1999-2000. The higher DAQ lifetime in 1999-2000 was due to recording correlated events only, rather than all triples, thus strongly reducing the load on the DAQ system. For the case of no oscillations, the combined efficiency of the trigger and selection cuts on neutrino interactions is about $18 \%$. The detector deadtime further reduces the efficiency to about $10 \%$ (the exact figure for each period being given in Table I). Experimental backgrounds may be naturally classified as uncorrelated and correlated, with uncorrelated backgrounds due to random coincidences between triple triggers within the delayed coincidence window, and correlated background due to events in which both subevents belong to the same process.

The dominant source of uncorrelated events is natural radioactivity. The inter-event time distribution for uncorrelated background events follows an exponential function with a time constant of $\sim 500 \mu \mathrm{s}$, as would be expected given the muon veto rate of $\sim 2 \mathrm{kHz}$ and the veto-dependent event selection requirements. This time dependence is slow com- pared to that of signal and correlated backgrounds, hence the uncorrelated background could be separated and studied by looking at long inter-event times.

The main source of correlated background are neutrons from muon spallation or capture. These events are mainly comprised of proton-neutron events in which a single neutron deposits its kinetic energy by scattering from protons and is then captured, and double neutron events in which two (typically thermal) neutrons from the same spallation event are captured in the detector.

Our analysis is based on 350.5 days of data taking, of which 242.2 days were at full power and the remainder at partial power with a reactor down for refueling. For the analysis we subdivided the data into eight periods. Four of the periods correspond to the four reactor refueling periods in which one reactor was off (off periods). Each of the remaining four periods (on periods) are constructed from intervals of full power data bracketing each refueling period. Table I shows the running time for each of the eight periods and the distance to the down reactor for each of the off periods.

The raw trigger rates for triples and correlated triples were approximately 50 and $1 \mathrm{~Hz}$, respectively. For 19992000 , the typical event rate after selection was $\sim 55 \mathrm{~d}^{-1}$ with all reactors at full power. Under the assumption of no oscillations, the efficiency after the trigger, deadtime, and event selection for detecting $\bar{\nu}_{e}$ 's above inverse beta decay threshold was $\sim 11 \%$; precise estimates of the efficiency period by period are listed in Table I. The observed event rate $N_{1}$ may be compared to an expected signal rate of $\sim 25 \mathrm{~d}^{-1}$ for no oscillations, implying a signal-to-noise ratio of $\sim 0.8$. The uncorrelated background event rate after selection was $\sim 7 \mathrm{~d}^{-1}$.

\section{A. Analysis with the "reactor power" method}

From Table I it is evident that the event rate is significantly lower during each refueling period. To investigate more quantitatively the correlation between event rates and reactor power, in Fig. 5 we plot the experimental rate corrected for efficiency and deadtime $R_{\text {expt }}$, against the calculated signal rate $R_{\text {calc }}$ expected for no oscillations. Only sta- 


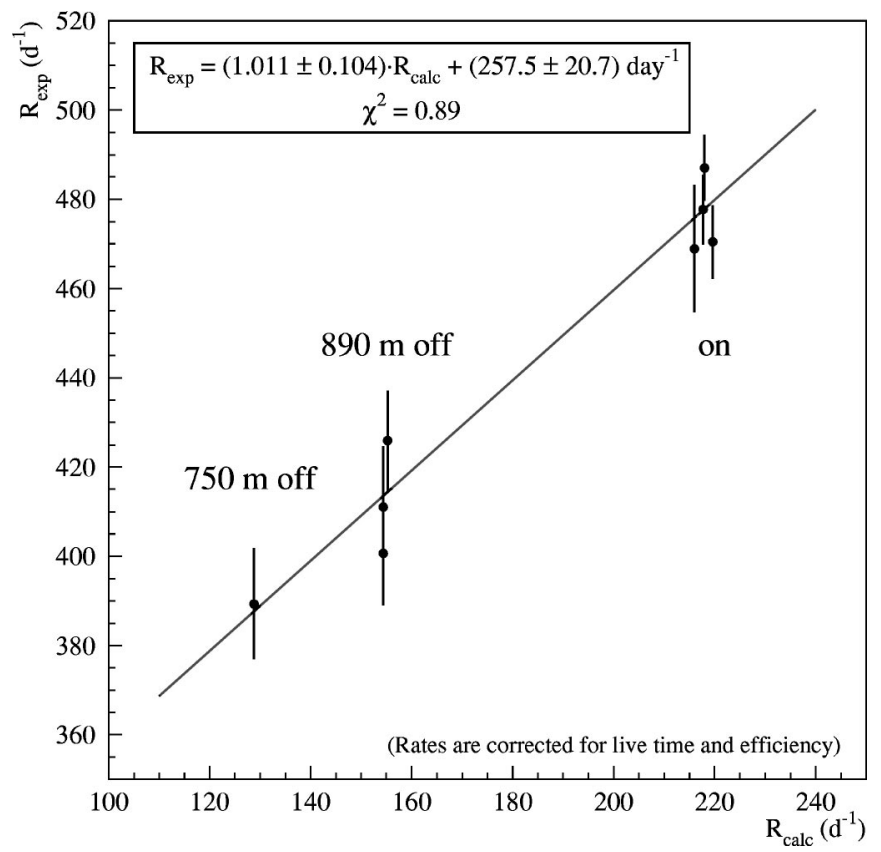

FIG. 5. The event rates $R_{\text {expt }}$ for different data taking periods, corrected for deadtime and neutrino detection efficiency, plotted vs the expected neutrino interaction rate $R_{\text {calc }}$ for no oscillations. Errors are statistical only. Points corresponding to data taking periods with same reactor power conditions should lie on top of each other. Also shown is the result, discussed in the text, for a linear fit to the data.

tistical uncertainties are indicated. If the data were consistent with no oscillations and the background were constant, then the points should lie along a straight line with unity slope. The $y$ intercept is equal to the rate of background interactions scaled by the ratio of the effective background detection efficiency to the neutrino detection efficiency. The data are in fact consistent with lying along a straight line. A linear fit to these data gives a slope of $1.011 \pm 0.104$ (stat.) and a $y$ intercept of $257.5 \pm 20.7$ (stat.) $\mathrm{d}^{-1}$. The reduced $\chi^{2}$ of the fit is 0.89 . Our data are therefore consistent with the hypothesis of no oscillations.

We have also analyzed the energy dependence of the neutrino interactions to see whether it is consistent with no oscillations as well. For each of the four pairs of on-off periods, one may subtract the event rate at partial power from the rate at full power. The resulting difference, after the small correction for the fuel burn-up has been made, is the contribution to the full power event rate from the neutrinos emitted by the reactor unit which was being refueled during the off period. Figure 6 shows the measured on-off event rate difference binned in the visible prompt (positron) energy. These data are not corrected for efficiency or deadtime. To make this plot, the weighted average of the four pairs of on-off periods is taken. Also shown are the corresponding expectations from Monte Carlo simulations for two scenarios: no oscillations and oscillations with parameters obtained from the best fit to the Kamiokande data [3]. The comparison of our data with Monte Carlo shows that the hypothesis of no oscillations is consistent not only with the measured event rate but also with the observed positron energy spectrum

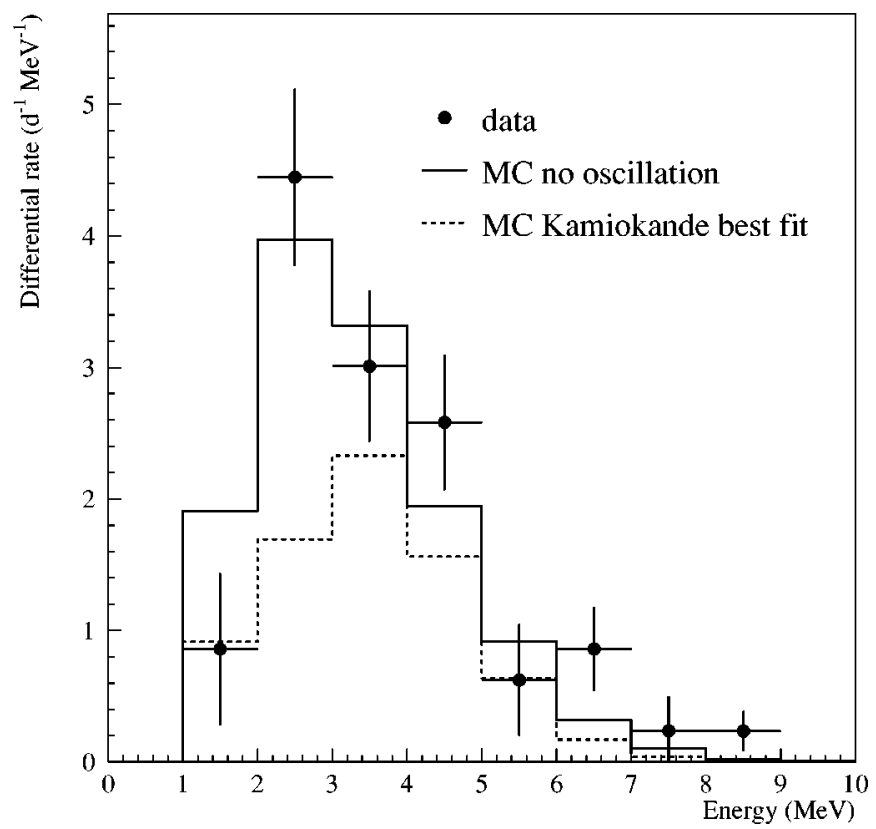

FIG. 6. The prompt energy spectrum after on-off subtraction averaged over the four pairs of on-off periods. The histograms show the corresponding expectations for no oscillations (solid line) and the Kamiokande best fit (dashed line).

( $\chi^{2} /$ d.o.f $=1.39$ for eight degrees of freedom), while it is not consistent with the rates implied by the Kamiokande best fit parameters $\left(\chi^{2} /\right.$ d.o.f $\left.=3.69\right)$.

To test our data for oscillation hypotheses throughout the $\Delta m^{2}-\sin ^{2} 2 \theta$ plane for two flavor mixing, a $\chi^{2}$ analysis using the "reactor power" changes was carried out. $\chi^{2}$ is defined as

$$
\chi^{2}=\sum_{i=1}^{8} \frac{\left(R_{\mathrm{exp}}^{i}-b g-\alpha \cdot R_{\mathrm{calc}}^{i}\right)^{2}}{\sigma_{i}^{2}}+\frac{(\alpha-1)^{2}}{\sigma_{\text {syst }}^{2}},
$$

where $R_{\text {expt }}^{i}$ is the observed rate for period $i, \quad b g$ is the background rate, $R_{\text {calc }}^{i}$ is the calculated rate for the period $i$ which depends on $\Delta m^{2}$ and $\sin ^{2} 2 \theta$, and $\alpha$ accounts for possible global normalization effects due to systematic uncertainties. $\sigma_{i}^{2}$ denotes the statistical uncertainty of each run period $i$, while $\sigma_{\text {syst }}=0.061$ is the systematic uncertainty discussed below (also see Table II). The quantity $b g$ is scaled

TABLE II. Contributions to the systematic error of the "reactor power" and "swap" analyses.

\begin{tabular}{lcc}
\hline \hline Error source & "Reactor power" $(\%)$ & "swap" $(\%)$ \\
\hline $\mathrm{e}^{+}$trigger efficiency & 2.0 & 2.0 \\
n trigger efficiency & 2.1 & 2.1 \\
$\bar{\nu}_{\mathrm{e}}$ flux prediction & 2.1 & 2.1 \\
$\bar{\nu}_{\mathrm{e}}$ selection cuts & 4.5 & 2.1 \\
Background variation & 2.1 & $\mathrm{~N} / \mathrm{A}$ \\
$\left(1-\epsilon_{1}\right) B_{\mathrm{pn}}$ estimate & $\mathrm{N} / \mathrm{A}$ & 3.3 \\
Total & $\mathbf{6 . 1}$ & $\mathbf{5 . 3}$ \\
\hline \hline
\end{tabular}


by 0.94 for the two periods in 1998 to account for the different trigger conditions in 1998 but is otherwise treated as constant. This scaling factor was determined from measuring how the rates for double neutron background events (the selection of which is described in Sec. IV B) and uncorrelated background events changed between 1998 and 1999.

To define the 90\% confidence level (C.L.) acceptance region for our data, we have followed the procedure suggested by Feldman and Cousins (FC) [22]. We implemented this procedure in two ways.

The first, called the Monte Carlo implementation, was realized as follows. A $\chi_{\text {best }}^{2}$ was determined by minimizing Eq. (1) with respect to $b g, \alpha, \Delta m^{2}$, and $\sin ^{2} 2 \theta$ for physically allowed values of $\Delta m^{2}$ and $\sin ^{2} 2 \theta$. We found the best fit to correspond to a null $\sin ^{2} 2 \theta$ with $\alpha=1.007$ (slightly unphysical but well within the statistically acceptable range) and a $\chi_{\text {best }}^{2}$ d.o.f. $=5.8 / 6$. The physical $\Delta m^{2}-\sin ^{2} 2 \theta$ plane was then subdivided into a fine grid. At each grid point, we minimized Eq. (1) with respect to $b g$ and $\alpha$ to obtain $\Delta \chi_{\text {data }}^{2}$ $\equiv \chi_{\text {data }}^{2}\left(\Delta m^{2}, \sin ^{2} 2 \theta\right)-\chi_{\text {best }}^{2}$. For determining whether the grid point was allowed at the $90 \%$ C.L., we simulated $10^{4}$ independent experiments at each grid point. The same $\chi^{2}$ minimization procedure was carried out for each simulated experiment as for the data to obtain $10^{4} \Delta \chi_{\mathrm{MC}}^{2}$ 's. These $\Delta \chi_{\mathrm{MC}}^{2}$ 's were sorted in increasing order to find $\Delta \chi_{\mathrm{c}}^{2}$, the value of $\Delta \chi^{2}$ greater than $90 \%$ of the $\Delta \chi_{\mathrm{MC}}^{2}$ 's. If $\Delta \chi_{\text {data }}^{2}$ $<\Delta \chi_{\mathrm{c}}^{2}$, the grid point was accepted.

The second way in which we implemented the FC procedure, called the raster scan, subdivides the two-dimensional grid in $\Delta m^{2}$ slices. For each value of $\Delta m^{2}$, Eq. (1) is minimized with respect to $b g, \alpha$, and $\sin ^{2} 2 \theta$. The value obtained for $\sin ^{2} 2 \theta$, without restricting the fit to the physically allowed range, is denoted $\left(\sin ^{2} 2 \theta\right)_{\text {best }}$ and its corresponding error is denoted $\sigma_{s i n}$. The best fit is slightly un-physical (but well within statistical errors); for $\Delta m^{2}=0.1 \mathrm{eV}^{2}$, $\left(\sin ^{2} 2 \theta\right)_{\text {best }} / \sigma_{\text {sin }}=-0.2$. The one-dimensional 90\% C.L. upper limit on $\sin ^{2} 2 \theta$ at the fixed value of $\Delta m^{2}$ is then given by $\alpha_{\mathrm{FC}} \sigma_{\text {sin }}$ where $\alpha_{\mathrm{FC}}$ is looked up from Table X of Ref. [22] for $x_{0} \equiv\left(\sin ^{2} 2 \theta\right)_{\text {best }} / \sigma_{\text {sin }}$.

While the raster scan method does not yield the global $\chi^{2}$ minimum in the $\Delta m^{2}-\sin ^{2} 2 \theta$ plane, it is computationally much faster. Checks have been carried out that the two methods for implementing the Feldman-Cousins procedure yield the same limits. For the purpose of determining the regions of parameter space excluded by our data, knowledge of the $\chi^{2}$ global minimum is not required. We have therefore used the raster scan method to obtain the exclusion curves reported in this paper.

The dashed curve in Fig. 7 shows the region of $\Delta m^{2}-\sin ^{2} 2 \theta$ plane excluded at the $90 \%$ C.L. by our data analyzed with the "reactor power" method. In the limit of large $\Delta m^{2}$, the range $\sin ^{2} 2 \theta>0.33$ is excluded; whereas in the limit of maximal mixing, the range $\Delta m^{2}>1.6$ $\times 10^{-3} \mathrm{eV}^{2}$ is excluded. We note that, in the limit of large $\Delta m^{2}$, the Monte Carlo method excludes the range $\sin ^{2} 2 \theta$ $>0.35$.

As already mentioned the independent analysis discussed in detail in Refs. $[1,2]$ was also improved and repeated for

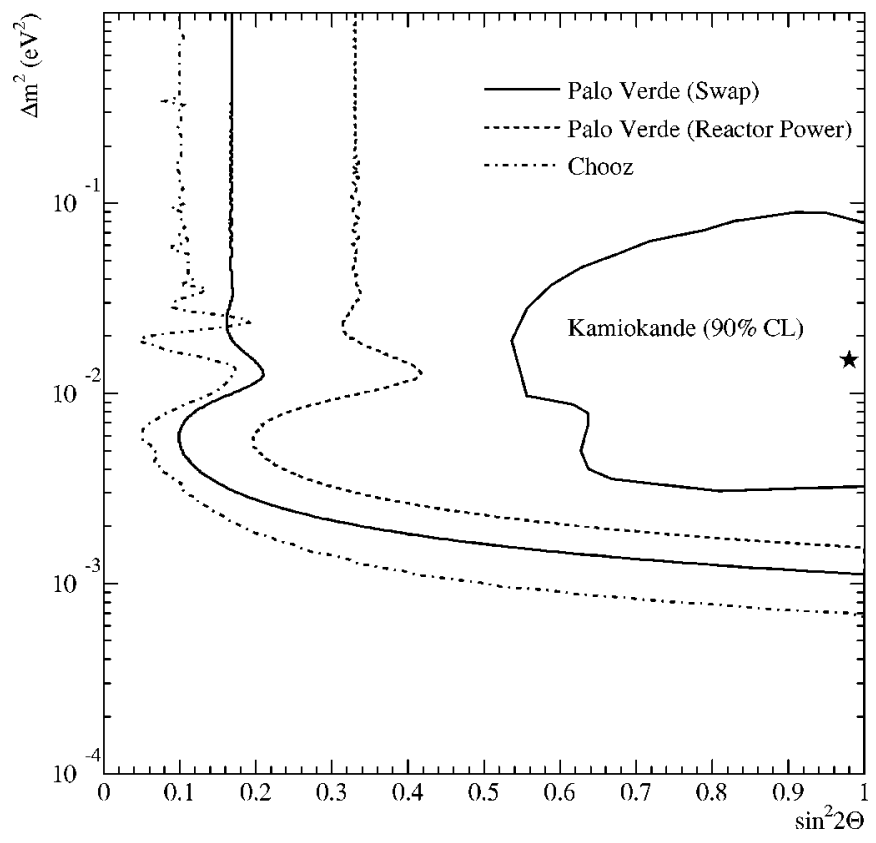

FIG. 7. Regions of $\Delta m^{2}-\sin ^{2} 2 \theta$ plane (two flavor oscillations) excluded at the $90 \%$ C.L. by "reactor power" analysis (dashed curve) and "swap" analysis (solid curve). Also shown are the Kamiokande allowed region and best fit (star) and the region excluded by the $\mathrm{CHOOZ}$ experiment $[6,7]$.

the full data set. In this case the "reactor power" analysis differs from the one described above in that the data are more finely binned by run rather than averaged by period. There were typically two runs per day. After combining short runs (runs with fewer than six neutrino candidates) with adjacent runs, 698 data points were obtained. A $\chi^{2}$ analysis identical in approach to that described above was carried out using a systematic error that in this case amounts to $6.9 \%$. Again, the best fit is slightly un-physical; for $\Delta m^{2}$ $=0.1 \mathrm{eV}^{2}\left(\sin ^{2} 2 \theta\right)_{\text {best }} / \sigma_{\text {sin }}=-0.5$. The $90 \%$ C.L. exclusion contour obtained in this analysis is very similar to the dashed curve in Fig. 7, but it is shifted toward smaller $\sin ^{2} 2 \theta$, with $\sin ^{2} 2 \theta>0.29$ excluded in the large $\Delta m^{2}$ limit. The shift in the exclusion boundary is consistent with small systematic differences expected between the two independent reconstructions and analyses.

\section{B. Analysis with the "swap" method}

The "swap" method, where the background is directly subtracted rather than using modulation of the reactor power, has substantially greater statistical power than the "reactor power" method. In addition, it has somewhat different systematics.

We briefly describe the method here; detailed descriptions have already been published $[1,2,23]$. Let $N_{1}$ be the event rate after applying the neutrino selection cuts described above. We then call $N_{2}$ the rate obtained by applying the positron cuts to the delayed sub-events and the neutron cuts to the prompt sub-events ("swapped" selection). The measurements of $N_{1}$ and $N_{2}$ are listed for each period in Table I. It is found that only about $20 \%$ of the neutrino signal cancels in the difference $N_{1}-N_{2}$, as determined from Monte Carlo 
simulation. At the same time the uncorrelated background and the double neutron component that dominates the correlated background cancel in the difference. We call $\left(1-\epsilon_{1}\right) B_{\mathrm{pn}}=\left(1-\epsilon_{\mathrm{sp}}\right) B_{\mathrm{pn}, \mathrm{sp}}+\left(1-\epsilon_{\mathrm{cap}}\right) B_{\mathrm{pn}, \text { cap }}$ the residual contribution to $N_{1}-N_{2}$, mainly due to the proton-neutron ("pn") component of the correlated background. Here the $\epsilon$ 's refer to the efficiency of the "swapped" selection for each channel [23], "sp" denotes neutron production by $\mu$ spallation (mainly in the laboratory walls), "cap" by muon capture (mainly in the water buffer) and " 1 ," maintaining the notation from our earlier papers, the total. While the capture process is well understood and can be reliably calculated with Monte Carlo simulations, the spallation is rather poorly known. The shape of the prompt energy spectrum for neutrons from spallation was obtained by generating neutrons in the laboratory walls according to several parametric models and passing them through the detector simulation and event selection. The set of parametric models spanned the range of uncertainty in our knowledge of the energy dependence of neutron production. The normalization was then determined by assuming that high-energy neutrino-like events, selected by replacing the cut on maximum hit energy $(<8 \mathrm{MeV})$ in the "positron" sub-event by the requirement that at least one prompt hit has an energy greater than $10 \mathrm{MeV}$, are due exclusively to spallation. The uncertainty on the energy spectrum, quantified by the dispersion between the different models, was taken into account in the systematic error. The term $B_{\text {pn,cap }}$ was found from the measured muon rate through the detector combined with the veto inefficiencies and the relatively well known total neutron production cross section and energy distribution by muon capture on oxygen. The systematic error is derived from the veto inefficiency.

The quantity $\left(1-\epsilon_{1}\right) B_{\mathrm{pn}}$ was estimated period by period, and the results are shown in Table I. The magnitude of (1 $\left.-\epsilon_{1}\right) B_{\mathrm{pn}}$ is small compared to the difference $N_{1}-N_{2}$, that is, the contribution from the proton-neutron component of the background largely cancels in the difference. Therefore, even though the systematic error on $\left(1-\epsilon_{1}\right) B_{\mathrm{pn}}$ is of order $100 \%$, the resulting contribution to the systematic error on the neutrino signal is only a few percent. In the sixth line of Table I for each run period we list the resulting background (assuming for the purpose of this illustration that the background efficiency is the same as for the signal, and correcting for the lifetime). The observed $\bar{\nu}_{\mathrm{e}}$ rate $\left(R_{\nu}\right)$, corrected for the lifetime and efficiency, and the expected neutrino rate $R_{\text {calc }}$ for no oscillations are also given in the table.

Similarly to the reactor power analysis, we have carried out a $\chi^{2}$ analysis to test our data for oscillation hypotheses throughout the two flavor oscillation $\Delta m^{2}-\sin ^{2} 2 \theta$ plane. The $\chi^{2}$ definition is

$$
\begin{aligned}
\chi^{2}= & \sum_{i=1}^{8} \frac{\left(N_{1, i}-N_{2, i}-\left(1-\epsilon_{1}\right) B_{\mathrm{pn}}-\alpha\left(R_{\text {calc }}^{1, i}-R_{\text {calc }}^{2, i}\right)\right)^{2}}{\sigma_{i}^{2}} \\
& +\frac{(\alpha-1)^{2}}{\sigma_{\text {syst }}^{2}},
\end{aligned}
$$

where $\sigma_{\text {syst }}$ for the "swap" method is estimated to be 0.053 as discussed below. The free parameters in this definition of the $\chi^{2}$ are $\Delta m^{2}, \sin ^{2} 2 \theta$, and $\alpha$. The Monte Carlo method gives $\chi_{\text {best }}^{2} /$ d.o.f. $=10.3 / 7$ for $\sin ^{2} 2 \theta$ consistent with zero and $\alpha=1.008$ (again, slightly un-physical but well within the statistical accuracy).

The region of parameter space excluded at the $90 \%$ C.L. by this analysis, based on the raster scan method, is indicated by the solid curve in Fig. 7. In the limit of large $\Delta m^{2}$, the range $\sin ^{2} 2 \theta>0.164$ is excluded; whereas in the limit of large mixing, the range $\Delta m^{2}>1.1 \times 10^{-3} \mathrm{eV}^{2}$ is excluded. We note that, in the limit of large $\Delta m^{2}$, the Monte Carlo method excludes the range $\sin ^{2} 2 \theta>0.162$, and gives an essentially identical exclusion curve.

\section{Test of the "swap" method}

As a further test of the "swap" method, we have investigated the energy dependence of $N_{1}$ and $N_{2}$. The measured energy dependence was compared to what would be expected on the basis of our assumptions about the signal and background. Were a significant source of background ignored or incorrectly treated, a discrepancy between data and expectation would result. To carry out this investigation, we assembled five samples of events:

$\bar{\nu}_{\mathrm{e}}$ : Inverse beta decay events were generated and simulated in the detector with normalization determined from the reactor powers, cross section, and number of target protons. No oscillations were assumed, as consistent with the outcome of the reactor power analysis.

Uncorrelated background: These events were selected from our data by inverting the spatial and temporal correlation requirements between the prompt and delayed subevents. The data sample was normalized to reproduce the event rate at large inter-event times.

$B_{\mathrm{pn}, \mathrm{sp}}$ : Neutrons produced by muon spallation in the laboratory walls were generated and passed through our detector simulation. As already mentioned, the sample was normalized by assuming that high-energy events satisfying the neutrino selection cuts are due to spallation.

$B_{\text {pn,cap }}$ : Neutrons produced by muon capture in water were generated and passed through the detector simulation. This data sample was normalized on the basis of the measured muon rate through the detector, the muon veto inefficiency, the fraction of muons stopping in the water, and the cross section for muon capture and neutron emission.

Double neutron: Double neutron events were selected from data by requiring a muon hit within $100 \mu$ s preceding the start of the event, and applying the neutron capture cuts to both the prompt and delayed sub-events. The sample was normalized so that-after application of the neutrino selection cuts-the combined five samples gave the measured total $N_{1}$ rate.

The five data samples were subjected to the neutrino event selection cuts $\left(N_{1}\right)$ and the swap event selection cuts $\left(N_{2}\right)$, respectively, and summed. The resulting energy spectra, with statistical uncertainties, are shown as histograms in Fig. 8. The expectation from the sum of the five samples is in good agreement with the data (points). Keeping in mind that only the overall normalization of the $N_{1}$ spectrum is not 

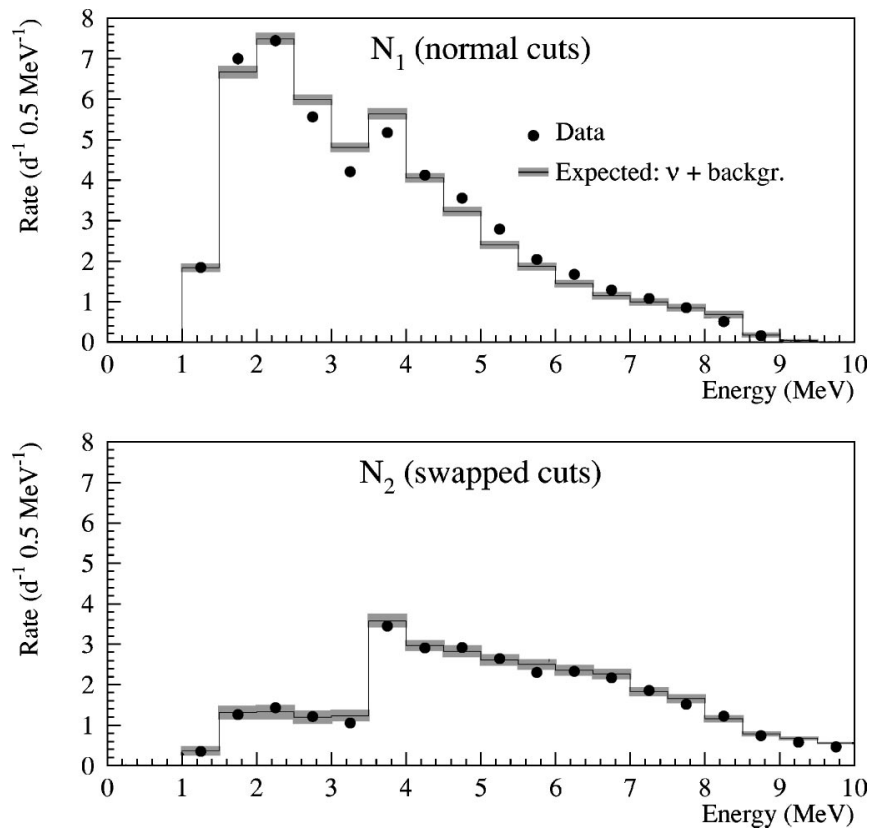

FIG. 8. The energy spectrum of events comprising $N_{1}$ and $N_{2}$. The points are measurements while the histogram shows the result of calculations described in the text. The shading indicates the statistical uncertainty in the calculation, while error bars for the measurements are too small to be visible.

independently determined, the validity of the "swap" analysis is solidly supported by this test.

\section{Systematic uncertainties}

The systematic uncertainty receives contributions from the detection efficiency and the flux calculation. In addition, the "reactor power method" suffers a systematic error from background variations with time, and the systematic uncertainty in the "swap" method has a contribution from the uncertainty in the estimate of $\left(1-\epsilon_{1}\right) B_{\mathrm{pn}}$. We have estimated the systematic uncertainty in the detection efficiency as follows.

$\bar{\nu}_{\mathrm{e}}$ selection cuts efficiency: The neutrino event selection cuts were varied randomly in the multi-dimensional cut space over a reasonable range. For each variation, the ratio of the observed number of events to that expected for no oscillations was calculated. The systematic error in the event selection efficiency was taken to be the rms of the variations in the ratio. The uncertainties in the definition of the energy scale are absorbed in this error component. The lower systematic uncertainty for the swap method is due to cancellation of some systematics in the difference $N_{1}-N_{2}$.

$\mathrm{e}^{+}$trigger efficiency: The systematic uncertainty in the $\mathrm{e}^{+}$ trigger efficiency is based on a comparison of simulated efficiencies with the measured efficiencies for the ${ }^{22} \mathrm{Na}$ calibration runs described above. To decouple uncertainties in the event selection efficiency from uncertainties in the trigger efficiency, loose cuts designed to have negligible inefficiency were applied to select ${ }^{22} \mathrm{Na}$ events for this analysis. The run-by-run comparison of the simulated and measured efficiencies was already shown in Fig. 3. Averaged over all runs, the efficiencies agree to $0.2 \%$ with a rms of $6.7 \%$. Grouping the runs by four calibration periods, the agreement was $1.3 \%$. Combining this with the $1.5 \%$ uncertainty in the activity of the source, the estimated systematic error is $2.0 \%$.

$n$ trigger efficiency: In an approach similar to that for estimating the systematic error in the $\mathrm{e}^{+}$trigger efficiency, we have used the Am-Be calibration runs described above to estimate the systematic uncertainty in the n trigger efficiency. The run-by-run comparison of measured versus simulated $n$ trigger efficiency was already shown in Fig. 3. The simulated efficiency is typically lower than the measured one. This difference is largely systematic, as manifested by the relatively small $\mathrm{rms}$ of $3.5 \%$ across the different calibration periods and positions. Averaged over runs, the difference between simulation and measurement is $2.1 \%$, which we assign as the systematic uncertainty for the $\mathrm{n}$ trigger efficiency.

The results for these sources of systematic error for the two analysis methods are listed in Table II. As explained in Sec. II B the systematic uncertainty in the $\bar{\nu}_{\mathrm{e}}$ flux is estimated to be $2.1 \%$.

The stability of background rates is a key assumption for the "reactor power" analysis. The actual level of background stability was estimated by comparing the average rate (lifetime corrected) during the full power periods to the average rate during the partial power periods for several background data samples: double neutrons, $B_{\mathrm{pn}}$, and uncorrelated background.

In addition, Michel electron events, present in the data due to the inefficiency of the veto detector, were used to track changes in the veto efficiency and, in particular, in the background due to neutron production by muon capture in water. These events are selected by requiring no activity in the muon detector, energy depositions in the prompt subevent consistent with a muon track, and a delayed subevent 5$20 \mu$ s later with an energy deposit of 10-70 MeV.

The Michel electron data sample was observed to have a rate stability better than $5 \%$ and all other data samples were found to be stable to better than $1 \%$. The rate variation for each background was normalized to its estimated contribution to the neutrino event rate and then divided by $12.9 \mathrm{~d}^{-1}$, the average difference in lifetime-corrected event rates between full power and partial power periods. Combined, the resulting ratios for the four backgrounds indicated a background instability of $2.1 \%$ relative to the signal. We thus take $2.1 \%$ as our estimate of the contribution to the systematic error in the "reactor power" analysis from background variations.

The contribution to the uncertainty in $\left(1-\epsilon_{\mathrm{sp}}\right) B_{\mathrm{pn}, \mathrm{sp}}$ from muon spallation in the walls was estimated from the spread in results from the four different models used to simulate neutron production, and was found to be $0.29 \mathrm{~d}^{-1}$. The estimated contribution to the uncertainty in $\left(1-\epsilon_{\text {cap }}\right) B_{\text {pn,cap }}$ from the veto counter inefficiency resulted to be $0.94 \mathrm{~d}^{-1}$. Thus the total systematic uncertainty on $\left(1-\epsilon_{1}\right) B_{\mathrm{pn}}$ amounted to $0.98 \mathrm{~d}^{-1}$. This result was lifetime corrected, and corresponded to $3.3 \%$ of the average lifetime-corrected value for $N_{1}-N_{2}$. The individual contributions are shown in Table II, and added in quadrature to obtain the total systematic error for each analysis method. 


\section{CONCLUSION}

The results presented here, based on nearly double the number of events of our previously published Palo Verde data, confirm the absence of $\bar{\nu}_{\mathrm{e}} \rightarrow \bar{\nu}_{x}$ oscillations for low energy reactor neutrinos. The excluded regions for our "reactor power" and "swap" methods are enlarged accordingly. For the "reactor power" method the new mixing angle limit is only slightly more restrictive than in our previous results. This is due to a small shift of the central value of the fit. A substantially larger exclusion region is obtained with the "swap" method, thanks to reduced systematics. In conclusion we find that the ratio of observed interaction rate to the one expected for no oscillations is $R_{\mathrm{obs}} / R_{\text {calc }}=1.01$ \pm 0.024 (stat) \pm 0.053 (syst). These final results are dominated by systematics errors.

Our measurements, along with those reported by $\mathrm{CHOOZ}$ $[6,7]$ and Super-Kamiokande [8], exclude two family $\nu_{\mu}-\nu_{e}$ mixing as being responsible for the atmospheric neutrino anomaly reported by Kamiokande [3].

\section{ACKNOWLEDGMENTS}

We would like to thank the Arizona Public Service Company for the generous hospitality provided at the Palo Verde plant. The important contributions of M. Chen, R. Hertenberger, K. Lou, and N. Mascarenhas in the early stages of this project are gratefully acknowledged. We are indebted to J. Ball, B. Barish, R. Canny, M. Dugger, A. Godber, J. Hanson, D. Michael, C. Peck, C. Roat, N. Tolich, A. Vital, and J. Winterton for their help. We also acknowledge the generous financial help from the University of Alabama, Arizona State University, California Institute of Technology, and Stanford University. Finally, our gratitude goes to CERN, DESY, Fermilab, LANL, LLNL, SLAC, and TJNAF, who at different times provided us with parts and equipment needed for the experiment. This project was supported in part by the Department of Energy and the National Science Foundation. One of us (J.K.) received support from the Hungarian OTKA fund, and another (L.M.) from the ARCS Foundation.
[1] F. Boehm et al., Phys. Rev. Lett. 84, 3764 (2000).

[2] F. Boehm et al., Phys. Rev. D 62, 072002 (2000).

[3] Y. Fukuda et al., Phys. Lett. B 335, 237 (1994).

[4] R. Becker-Szendy et al., Phys. Rev. Lett. 69, 1010 (1992).

[5] E. Peterson et al., Nucl. Phys. B (Proc. Suppl.) 77, 111 (1999).

[6] M. Apollonio et al., Phys. Lett. B 466, 415 (1999); Phys. Rev. D 61, 012001 (2000).

[7] M. Apollonio et al., Phys. Lett. B 420, 397 (1998).

[8] Y. Fukuda et al., Phys. Rev. Lett. 81, 1562 (1998).

[9] A. G. Piepke, S. W. Moser, and V. M. Novikov, Nucl. Instrum. Methods Phys. Res. A 432, 392 (1999).

[10] G. Gratta et al., Nucl. Instrum. Methods Phys. Res. A 400, 456 (1997).

[11] A. A. Hahn et al., Phys. Lett. B 218, 365 (1989).

[12] K. Schreckenbach et al., Phys. Lett. 160B, 325 (1985).
[13] P. Vogel et al., Phys. Rev. C 24, 1543 (1981).

[14] P. Vogel and J. F. Beacom, Phys. Rev. D 60, 053003 (1999).

[15] G. Zacek et al., Phys. Rev. D 34, 2621 (1986).

[16] B. Achkar et al., Nucl. Phys. B434, 503 (1995).

[17] Y. Declais et al., Nucl. Phys. B434, 503 (1995), and references therein.

[18] R. Brun et al., "GEANT 3," CERN DD/EE/84-1 (revised), 1987.

[19] P. A. Aarnio et al., "FLUKA user's guide," TIS-RP-190, CERN, 1990.

[20] T. A. Gabriel et al., ORNL/TM-5619-mc, April 1977.

[21] R. L. Craun and D. L. Smith, Nucl. Instrum. Methods 80, 239 (1970).

[22] G. J. Feldman and R. D. Cousins, Phys. Rev. D 57, 3873 (1998).

[23] Y. F. Wang et al., Phys. Rev. D 62, 013012 (2000). 